Pecvnia, Monográfico (2008), pp. 129-146

\title{
Análisis de los factores determinantes de la eficacia organizativa desde la dirección estratégica de recursos humanos
}

Nuria González Álvarez

nuria.gonzalez@unileon.es

Universidad de León

Organización de Empresas

Fac. de Ciencias Económicas y Empresariales

Campus de Vegazana, $\mathrm{s} / \mathrm{n}$

24071 León (España)

La dirección estratégica de recursos humanos enfatiza la influencia de las prácticas de recursos humanos de alto compromiso sobre la eficacia organizativa. El objetivo de este trabajo es contrastar como el empleo de un conjunto de prácticas de recursos humanos de alto compromiso en el seno de una organización influye positivamente en los resultados que ésta consigue. Para ello, se dispone de una muestra de 258 empresas manufactureras españolas. Los resultados apuntan que la utilización de dichas prácticas de recursos humanos tiene un impacto positivo sobre los rendimientos corporativos. De esta forma, este trabajo aporta evidencia empírica para la relación entre las prácticas de recursos humanos de alto compromiso consideradas
Strategic human resource management highlights the influence of the high commitment human resource practices on firm performance. The aim of this study is to analyse how the use of high commitment human resources practices influences, in a positive way, on the firm performance. For this purpose, a sample of 258 Spanish manufacturing companies has been used. The results indicate that the use of the said human resources practices have a positive impact on corporate performance. In this way, the main implication of this study is that provides empirical evidence for Spanish firms of the relationship between high involvement human resource practices and 
de forma global y el rendimiento organizativo para el caso de una gran muestra de empresas españolas pertenecientes a todos los sectores de actividad industrial, lo cual supone una avance importante en la investigación sobre recursos humanos en España donde existe una laguna importante de trabajos empíricos que aborden esta cuestión.

Palabras clave: prácticas de recursos humanos de alto compromiso, resultados corporativos. performance. This represents a significant advance in human resources research in Spain, where there is a significant gap in empirical work studying this subject.

Key words: high commitment human resource practices, firm performance.

\section{INTRODUCCIÓN}

Tanto investigadores como directivos del área de recursos humanos han sugerido que los recursos humanos son determinantes clave de los resultados empresariales (Barney y Wright 1998: 31). En la literatura de los últimos años se han puesto de manifiesto las implicaciones que las políticas de recursos humanos pueden tener sobre algunos de los resultados empresariales más relevantes. Así, existen investigaciones que relacionan las prácticas de dirección de recursos humanos con la marcha de los trabajadores (Arthur 1994; Guthrie 2001), con la productividad (Ichniowski, Shaw y Prennushi 1997; MacDuffie 1995; Guthrie 2001; Chen Liaw y Lee 2003), con los beneficios financieros (Delery y Doty 1996), con la supervivencia (Welbourne y Andrews 1996), con el valor de la empresa (Huselid 1995) o con el desempeño organizativo (Bae y Lawler 2000; Chang y Huang 2005).

Tomando como marco general la dirección estratégica de recursos humanos, numerosos trabajos han planteado la existencia de una serie de prácticas de recursos humanos, llamadas de alto rendimiento o de compromiso que contribuyen a que la empresa mejore de manera significativa sus resultados (e.g. Kochan y Osterman 1994; Lawler 1992, 1996; Levine 1995; Huselid 1995; Guthrie 2001). El objetivo de estas prácticas es mejorar las capacidades, el compromiso y la productividad de los empleados de una empresa (Datta, Guthrie y Wright 2005) consiguiendo que tomen como propios los objetivos de esta última.

El objetivo de este trabajo es contrastar como el empleo de un conjunto de prácticas de recursos humanos de alto compromiso en el seno de una organización influye positivamente en los resultados que ésta consigue. Serán aquellas organizaciones que apliquen las prácticas a las que nos referíamos en el párrafo anterior las que vean cómo sus resultados 
van mejorando con mayor intensidad. El ámbito de estudio es la industria española, en concreto se dispone de una muestra de 258 empresas manufactureras españolas. Así se intentará comprobar si las conclusiones obtenidas sobre la cuestión objeto de estudio para otros contextos geográficos como Estados Unidos (e.g. Huselid 1995; Whitener 2001), Nueva Zelanda (e.g. Guthrie 2001), Irlanda (e.g. McCartney y Teague 2004), Corea (e.g. Bae y Lawler 2000) y Taiwán (e.g. Chang y Huang 2005) se reproducen también para el caso español. Por tanto, este trabajo aporta evidencia empírica para la relación entre las prácticas de recursos humanos de alto compromiso consideradas de forma global y el rendimiento organizativo para el caso de una gran muestra de empresas españolas pertenecientes a todos los sectores de actividad industrial, lo cual supone una avance importante en la investigación sobre recursos humanos en España donde existe una laguna importante de trabajos empíricos que aborden esta cuestión.

Con este propósito, en el siguiente apartado se propone un marco teórico y una hipótesis a contrastar. Posteriormente, se describe la metodología utilizada así como los resultados obtenidos. Finalmente, el último apartado recoge las principales conclusiones que de este trabajo pueden extraerse así como sus principales limitaciones y futuras líneas de investigación.

\section{MARCO TEÓRICO E HIPÓTESIS A CONTRASTAR}

Recientemente el interés en el campo de la dirección de recursos humanos se ha intensificado ya que algunos investigadores han otorgado especial relevancia al papel del personal de la empresa como fuente única de ventaja competitiva (Wright y McMahan 1992; Pfeffer 1994, 1998). La cuestión que subyace es si las empresas van a ser capaces, y de qué forma, de capitalizar la fuente de ventaja competitiva que constituyen sus recursos humanos. Esta área creciente de investigación que resalta la importancia de los recursos humanos se ha denominado dirección estratégica de recursos humanos ya que enfatiza el papel estratégico de la dirección de recursos humanos en la consecución de los objetivos empresariales. Wright and McMahan definen la dirección estratégica de recursos humanos como el "conjunto de actividades planificadas de recursos humanos que permiten a la empresa alcanzar sus objetivos" (1992: 298). 
Como consecuencia del reconocimiento del potencial de los activos humanos como fuente de ventaja competitiva, muchos académicos han utilizado la teoría de recursos y capacidades para entender el papel que juegan los recursos humanos en las organizaciones. Por ejemplo, Wright, McMahan y McWilliams (1994) analizan la forma en la que los recursos humanos pueden constituir una fuente de ventaja competitiva sostenible. Estos autores sostienen que los recursos humanos pueden proporcionar una ventaja competitiva sostenible cuando cumplen cuatro requisitos. En primer lugar, los recursos deben añadir valor a los procesos de producción organizativos y en este contexto, los niveles de rendimiento individual pueden ser importantes. En segundo lugar, las destrezas que la empresa necesita deben ser raras. Al respecto, los recursos humanos valiosos pueden ser considerados como raros en el sentido que el conocimiento, las destrezas y las habilidades humanas están normalmente distribuidas en la población. Como tercer criterio, estos autores plantean que la inversión en capital humano que los empleados de una empresa representan, no es fácilmente imitable. En este sentido, es obvio que los recursos humanos no están sujetos al mismo grado de imitabilidad que los equipos o las infraestructuras. Además, el personal de una organización pueden contribuir a la disminución de la probabilidad de réplica debido a la diferencias de tipo cualitativo que pueden existir entre los empleados de una empresa y los de sus competidores. Finalmente, los recursos humanos de una empresa no serán fácilmente sustituibles por avances tecnológicos u otros sustitutos si están proporcionando una ventaja competitiva sostenible. Por todo lo anterior, el trabajo de Wright y McMahan (1992) pone de relevancia la importancia de los recursos humanos en la creación de la ventaja competitiva empresarial.

Según Delery y Shaw (2001), al menos dos características diferencian a la dirección estratégica de recursos humanos de la dirección de recursos humanos tradicional. En primer lugar, los estudios sobre dirección estratégica de recursos humanos se centran en explicar el papel estratégico que los recursos humanos pueden jugar a la hora de aumentar la eficacia organizativa. Una segunda característica es el nivel de análisis. Las prácticas de recursos humanos han sido analizadas tradicionalmente desde un enfoque individual (Katz, Kochan y Gobeille 1983; Holzer 1987; Morishima 1991; Bartel 1994). En constaste la investigación en dirección estratégica de recursos humanos se ha llevado a cabo a nivel de negocio o organizativo. Como reflejo de esta orientación, los trabajos recientes se han centrado en los sistemas de recursos humanos de alto rendimiento, 
un término usado para referirse a un sistema de prácticas de recursos humanos diseñadas para estimular las habilidades de los empleados, su compromiso y su productividad con el fin de convertir a estos empleados en una fuente de ventaja competitiva (Lawler 1992, 1996; Levine 1995; Pfeffer 1998; Datta, Guthrie y Wright 2005).

Las prácticas de recursos humanos de alto compromiso o alta implicación crean una fuerza de trabajo que está motivada y altamente comprometida con los destinos de la organización. Estas prácticas incluyen, entre otras, la participación de los trabajadores, la preocupación por su formación y el cuidado de los procesos de formación. Frente a aquellas prácticas más tradicionales que lo que buscan es controlar a los trabajadores para que éstos desarrollen unos comportamientos previamente definidos con exactitud (Walton 1985), mediante la dirección de personal de alto compromiso se persigue mejorar las capacidades del trabajador y aumentar su motivación. Según Huselid (1995: 635):

las prácticas de recursos humanos de alto compromiso pueden mejorar los conocimientos, destrezas y habilidades tanto de los empleados actuales como potenciales de una empresa aumentado su motivación (...) y fomentando la retención de empleados.

Así, existen numerosos estudios en la literatura que relacionan dichas prácticas con distintas variables empresariales como la marcha de los trabajadores (Arthur 1994; Huselid 1995; Guthrie 2001), con la productividad (Ichniowski, Shaw y Prennushi 1997; MacDuffie 1995; Huselid 1995), con los beneficios financieros (Delery y Doty 1996), con la supervivencia (Welbourne y Andrews 1996), con el valor de la empresa (Huselid 1995; Huselid y Becker 1997) o con el desempeño organizativo (Delaney y Huselid 1996; Bae y Lawler 2000).

Tomando como referencia estos trabajos, sobretodo los dos últimos, puede establecerse que las prácticas de recursos humanos de alto compromiso ejercen un efecto positivo sobre los rendimientos de las empresas, tal y como recoge la siguiente hipótesis:

$\mathrm{H}$ : La utilización por parte de una empresa de prácticas de recursos humanos que requieran una alta participación o un alto compromiso de sus empleados tiene una influencia positiva sobre los resultados que dicha organización alcance. 


\section{METODOLOGÍA}

\subsection{Muestra}

La muestra de empresas que se ha empleado para contrastar la hipótesis propuesta ha sido elaborada a partir del directorio Duns 50.000 de principales empresas españolas en su edición de 2001. El proceso de selección y recogida de información fue el siguiente. En primer lugar se limitó la muestra exclusivamente a las empresas manufactureras (con código SIC comprendido entre 20 y 39 ) de tamaño mediano o grande (con un volumen de ventas superior a los 20 millones de euros en 1999). Con ambos criterios se buscaba garantizar que las empresas a estudiar hubiesen desarrollado un cierto número de prácticas de recursos humanos. De esta forma se obtuvieron 1967 empresas que cumpliesen simultáneamente ambos criterios.

Tabla 1: Descripción de la muestra por sector de actividad

\begin{tabular}{|c|c|c|c|}
\hline \multicolumn{2}{|c|}{ Código SIC Sector de actividad } & \multirow{2}{*}{$\frac{\text { Empresas }}{42}$} & \multirow{2}{*}{$\frac{\text { Porcentaje }}{16,3}$} \\
\hline 20 & Industria alimentaria & & \\
\hline 21 & Fabricantes de tabaco & 0 & 0 \\
\hline 22 & Industria textil & 9 & 3,5 \\
\hline 23 & Prendas confeccionadas & 2 & 0,8 \\
\hline 24 & Industria de la madera & 2 & 0,8 \\
\hline 25 & Mueble y mobiliario & 0 & 0 \\
\hline 26 & Papel y derivados & 5 & 1,9 \\
\hline 27 & Editorial- artes graficas & 9 & 3,5 \\
\hline 28 & Productos químicos & 37 & 14,3 \\
\hline 29 & Petróleo y sus derivados & 1 & 0,4 \\
\hline 30 & Productos de goma y plástico & 12 & 4,7 \\
\hline 31 & Cuero y derivados & 2 & 0,8 \\
\hline 32 & Productos de piedra, arcilla, vidrio y hormigón & 22 & 8,5 \\
\hline 33 & Siderurgia & 10 & 3,9 \\
\hline 34 & Fabricación de metal excepto maquinaria y equipos de transporte & 16 & 6,2 \\
\hline 35 & Maquinaria & 27 & 10,5 \\
\hline 36 & Maquinaria eléctrica y electrónica & 21 & 8,1 \\
\hline 37 & Equipos de transportes & 30 & 11,6 \\
\hline 38 & Instrumentos de medida, análisis, control, fotografía, óptica y relojes & 6 & 2,3 \\
\hline \multirow[t]{2}{*}{39} & Fabricantes diversos & 5 & 1,9 \\
\hline & Total & 258 & 100 \\
\hline
\end{tabular}

En segundo lugar, como la información que proporciona el mencionado directorio era insuficiente para el desarrollo de la investigación, se recurrió al envío de un cuestionario a cada una de las 1.967 empresas. El cuestionario iba dirigido al director general de la empresa (CEO), ya que se consideró que era la persona más cualificada para responder a las 
preguntas formuladas y quién más fácil acceso tenía a la información solicitada. De esta forma, durante el período de recogida de datos se recibieron 258 cuestionarios válidos, lo que supuso un error muestral de $\pm 5,80 \%$ con un nivel de confianza de un $95 \%$. Las Tablas 1 y 2 representan una descripción de la muestra por sector de actividad y número de empleados.

Tabla 2: Descripción de la muestra por número de empleados

\begin{tabular}{lcc}
\hline Número de empleados & Empresas & Porcentaje \\
\hline $0-100$ & 54 & 20,9 \\
\hline $101-250$ & 93 & 36,0 \\
\hline $251-500$ & 66 & 25,6 \\
\hline $501-1000$ & 22 & 8,5 \\
\hline $1000-5000$ & 20 & 7,8 \\
\hline 5.000 & 3 & 1,2 \\
\hline Total & 258 & 100 \\
\hline
\end{tabular}

\subsection{Medidas de las variables}

En referencia a la forma de hacer operativas las variables incluidas en esta investigación, como medida de las políticas de recursos humanos de alto compromiso se utilizaron veintisiete indicadores extraídos de las publicaciones recientes sobre el tema (Cutcher-Gershenfeld 1991; Arthur 1992, 1994; Kochan y Osterman 1994; Huselid 1995; MacDuffie 1995; Delaney y Huselid 1996; Delery y Doty 1996; Bae y Lawler 2000). En el Anexo 1 se muestran estos indicadores.

Con objeto de resumir los datos obtenidos para las políticas de recursos humanos de alto compromiso, se llevó a cabo un análisis factorial según la técnica de componentes principales y, con el fin de obtener unos resultados más fácilmente interpretables, se optó por realizar una rotación de factores mediante el método de normalización de varimax con Kaiser. La Tabla 3 expone la matriz de componentes rotados así como las comunalidades, los autovalores iniciales y el porcentaje de varianza que explica cada componente. En base a esta matriz puede extraerse las siguientes conclusiones:

1. En el caso del primer factor, se puede observar como los indicadores relevantes son aquellos que hacen referencia al grado de motivación que poseen los empleados, a la cantidad de información que éstos comparten y a la presencia en la empresa 
de un clima de alta cooperación y confianza. Así, estas variables parecen implicar un cierto buen ambiente en la empresa, ya que los empleados se sienten motivados y el clima reinante es favorable. Por estas razones, el nombre asignado a este factor fue AMBIENTE.

Tabla 3: Políticas de recursos humanos. Matriz de componentes rotados y comunalidades

\begin{tabular}{lcccccccc}
\hline ITEMS & \multicolumn{7}{c}{ COMPONENTE } & \multicolumn{1}{c}{ COM } \\
\cline { 2 - 7 } & 1 & 2 & 3 & 4 & 5 & 6 & 7 & \\
\hline SEL1 & 0,153 & 0,472 & $-0,040$ & 0,233 & 0,626 & 0,160 & $-0,052$ & 0,72 \\
\hline SEL2 & 0,116 & 0,500 & $-0,040$ & 0,167 & 0,575 & 0,218 & $-0,001$ & 0,67 \\
\hline SEL3 & 0,170 & 0,170 & $-0,009$ & 0,101 & 0,744 & $-0,030$ & $-0,082$ & 0,63 \\
\hline SEL4 & 0,068 & 0,046 & 0,110 & 0,199 & 0,795 & 0,072 & 0,128 & 0,71 \\
\hline FORM1 & 0,178 & 0,705 & 0,257 & 0,204 & 0,207 & $-0,018$ & 0,142 & 0,70 \\
\hline FORM2 & 0,183 & 0,701 & 0,324 & 0,053 & 0,278 & 0,005 & 0,186 & 0,74 \\
\hline FORM3 & 0,240 & 0,608 & 0,409 & 0,121 & 0,145 & 0,010 & 0,111 & 0,64 \\
\hline FORM4 & 0,365 & 0,598 & $-0,114$ & 0,244 & $-0,058$ & $-0,040$ & $-0,054$ & 0,57 \\
\hline RETRI1 & 0,101 & 0,048 & 0,154 & 0,695 & 0,303 & 0,071 & $-0,108$ & 0,63 \\
\hline RETRI2 & 0,033 & 0,122 & $-0,016$ & 0,818 & 0,180 & 0,078 & 0,040 & 0,72 \\
\hline RETRI3 & 0,271 & 0,030 & 0,161 & 0,649 & 0,018 & 0,008 & 0,092 & 0,53 \\
\hline RETRI4 & 0,216 & 0,165 & 0,385 & 0,157 & 0,087 & 0,091 & $-0,427$ & 0,45 \\
\hline RETRI5 & 0,108 & $-0,118$ & 0,013 & 0,102 & 0,071 & 0,766 & 0,094 & 0,64 \\
\hline REND1 & 0,189 & 0,268 & 0,028 & 0,683 & 0,102 & 0,231 & 0,075 & 0,64 \\
\hline REND2 & 0,124 & 0,123 & 0,205 & 0,148 & 0,084 & 0,741 & 0,053 & 0,65 \\
\hline PUEST1 & 0,118 & 0,178 & 0,110 & 0,196 & 0,330 & 0,086 & 0,616 & 0,59 \\
\hline PUEST2 & 0,135 & 0,045 & 0,054 & 0,011 & $-0,119$ & 0,129 & 0,739 & 0,60 \\
\hline MOTI1 & 0,736 & 0,035 & 0,000 & 0,072 & 0,140 & 0,259 & 0,231 & 0,69 \\
\hline MOTI2 & 0,806 & 0,178 & 0,120 & 0,248 & 0,166 & 0,140 & 0,073 & 0,81 \\
\hline MOTI3 & 0,738 & 0,346 & 0,180 & 0,176 & 0,103 & 0,072 & $-0,153$ & 0,77 \\
\hline MOTI4 & 0,503 & 0,247 & 0,501 & 0,302 & 0,067 & 0,089 & 0,023 & 0,70 \\
\hline OTRS1 & 0,661 & 0,214 & 0,333 & 0,086 & 0,184 & $-0,109$ & 0,225 & 0,70 \\
\hline OTRS2 & 0,190 & $-0,005$ & 0,761 & 0,020 & 0,112 & $-0,017$ & $-0,005$ & 0,63 \\
\hline OTRS3 & 0,129 & 0,210 & 0,780 & 0,140 & $-0,072$ & 0,135 & $-0,009$ & 0,71 \\
\hline OTRS4 & 0,043 & 0,380 & 0,652 & $-0,051$ & $-0,056$ & 0,140 & 0,150 & 0,62 \\
\hline OTRS5 & 0,631 & 0,334 & 0,386 & 0,111 & 0,028 & 0,054 & $-0,094$ & 0,68 \\
\hline OTRS6 & 0,136 & 0,622 & 0,223 & 0,000 & 0,209 & $-0,031$ & $-0,084$ & 0,51 \\
\hline \%Varian & 31,86 & 8,82 & 6,74 & 5,30 & 5,06 & 4,10 & 3,40 & 65,3 \\
\hline Autoval. & 8,60 & 2,40 & 1,82 & 1,43 & 1,37 & 1,11 & 0,92 & \\
\hline & & & & & & & &
\end{tabular}

1 Los ítems de esta columna representan los indicadores utilizados para medir las políticas de recursos humanos de alto compromiso. El contenido de estos indicadores se detalla en el Anexo 1. 
2. En el segundo factor destacan los ítems referidos a los procesos de formación de empleados llevados a cabo por la empresa así como a la preocupación por las condiciones de seguridad de sus puestos de trabajo. Esto parece querer decir que la empresa se preocupa por sus empleados, sobretodo a nivel de formación. Por ello, este factor fue denominado FORMACIÓN.

3. Sobre el tercer factor cargan alto ítems como los referentes a la existencia de mecanismos y procedimientos destinado a aumentar la motivación de sus trabajadores en el seno de la empresa y a la presencia de grupos de solución de problemas así como la existencia de mecanismos de apoyo a las nuevas ideas (círculos de calidad y buzones de sugerencias). Así, este factor parece implicar la existencia de mecanismos que apoyen a la innovación y a la creatividad. Por ello, a este factor se le asignó el nombre de APOYO A LA INNOVACIÓN.

4. El cuarto factor viene representado por los indicadores referidos a las políticas de retribuciones llevadas a cabo por la empresa y a la evaluación del rendimiento en función de los resultados. Por ello, parece que trata de recoger el sistema de compensación existente en la empresa sobretodo basado en resultados. Por este motivo, este factor fue denominado como RETRIBUCIÓN BASADA EN RESULTADOS.

5. El factor que aparece en quinto lugar está integrado únicamente por los ítems que hacen referencia a los procesos de selección seguidos por la empresa por lo que recibió el nombre de SELECCIÓN.

6. En el caso del sexto factor se puede observar como los ítems relevantes son los referidos a la amplitud de salarios en un mismo puesto de trabajo y a la evaluación del rendimiento en función de la conducta. Así, parece querer mostrar una política de retribución de la empresa que descansa en la evaluación del rendimiento en términos de conducta, de ahí la variedad de salarios en un mismo puesto de trabajo. Por ello, este factor fue etiquetado como RETRIBUCIÓN BASADA EN CONDUCTA.

7. Finalmente, el séptimo y último factor está integrado por los indicadores referentes a un diseño amplio de puestos de trabajo, es decir, que los puestos de trabajo de la empresa incluyan gran variedad de tareas y exista rotación sobre ellos. Este factor fue denominado DISEÑO DE PUESTOS. 
Terminado el resumen y reducción de datos referentes a los indicadores que medían la política de recursos humanos de alto compromiso seguida por la empresa, se guardaron, mediante el método de regresión, las puntuaciones factoriales para cada uno de los factores hallados. A continuación, siguiendo a Youndt, Snell, Dean y Lepak (1996), Bae y Lawler (2000), Richard y Johnson (2001) y Guthrie (2001) se construyó un índice para las prácticas de recursos humanos de alto compromiso, basado en las puntuaciones de cada empresa para cada factor. En este sentido, se creó una nueva variable, prácticas de recursos humanos de alto compromiso, que podía tomar ocho valores posibles. Con el fin de distinguir si una empresa usa o no un factor particular de prácticas, se compara la puntuación de la empresa para ese factor con la puntuación media de todas las empresas de la muestra para ese factor. Dado que la media para cada factor es 0 ya que los factores son el resultado de un análisis factorial, la nueva variable toma valor 0 cuando la empresa no presenta ninguna puntuación positiva en ninguno de los siete factores en los cuales la política de recursos humanos de alto compromiso de la empresa fue dividida, lo que significa que la empresa no utiliza los sistemas de recursos humanos de alto compromiso de forma más intensiva que el resto de empresas. Por otro lado, la nueva variable toma valor 7 cuando la empresa presenta puntuaciones positivas en todos los factores de la política de recursos humanos de alto compromiso, lo que indica que la empresa utiliza las prácticas de recursos humanos de alto compromiso más intensivamente que el resto de empresas. Por tanto, un alto valor de la variable prácticas de recursos humanos de alto compromiso indica un uso intensivo de prácticas de recursos humanos de alto compromiso. Por el contrario, valores pequeños de esa variable indican un uso menos intensivo de dichas prácticas.

El uso de una medida única para las prácticas de recursos humanos de alto compromiso está en consonancia con los argumentos propuestos por Becker y Huselid (1998) quienes están de acuerdo con la práctica extendida en la literatura de que un índice derivado de un trabajo empírico previo es la medida más apropiada para un sistema de recursos humanos ya que dicha única medida recoge la idea de sistema de recursos humanos como activo estratégico.

Respecto al rendimiento empresarial señalar que éste se hizo operativo mediante dos medidas que también se recogen en el Anexo 1. En primer lugar, una medida subjetiva que incluía tanto indicadores económico-financieros como socio-organizativos debido a que sólo la 
consideración conjunta de todas ellos permite valorar el éxito de una organización (Robbins 1990). En consecuencia, siguiendo a Naman y Slevin (1993) se construyeron dos escalas de ítems. El objetivo de la primera escala era que los directivos valorasen la importancia otorgada a los indicadores propuestos. Con la segunda escala, se perseguía que los directivos expresasen el grado de satisfacción obtenido durante el último ejercicio en cuanto a sus expectativas con respecto a dichos indicadores. Posteriormente, se calculó una media ponderada de las puntuaciones de satisfacción otorgada por los directivos a los nueve indicadores, utilizando las puntuaciones de importancia como ponderaciones.

Adicionalmente, se consiguieron datos de los resultados financieros de las empresas, los cuales se extrajeron del directorio Duns. Concretamente se escogió la rentabilidad económica (ROA), ya que proporciona mayor estabilidad año a año que otras medidas financieras (Hill, Hitt y Hoskisson 1992) y continúa teniendo gran aceptación en la literatura (Wiersema y Bantel 1993; Balinga, Moyer y Rao 1996). Así, se tomaron datos de la rentabilidad económica alcanzada por las empresas para los tres últimos años publicados. Posteriormente se calculó la media aritmética de las tres medidas con el fin de eliminar la posibilidad de existencia de de resultados extraordinarios, siguiendo el procedimiento propuesto por King y Zeithaml (2001).

Aunque las medidas objetivas de resultados evidentemente propiedades deseables, se escogió utilizar la medida subjetiva ya que Delaney y Huselid (1996) observan que en varios estudios existen relaciones fuertes entre ambos tipos de medidas y adicionalmente el resto de variables se midieron a través de medidas de este tipo. Sin embargo, se utilizan los datos financieros recogidos (ROA) para contrastar la validez de la medida subjetiva, siguiendo los consejos de Bae y Lawler (2000). La correlación obtenida entre la rentabilidad económica y la medida subjetiva es de 0,34 $(\mathrm{p}<0,05)$. Dado que dicha correlación es estadísticamente significativa apoya la validez general de la medida subjetiva propuesta.

Adicionalmente, se seleccionaron algunas variables de control que se consideraba que podían estar relacionadas con algunas de las variables implicadas en el estudio. En este sentido se incluyeron como variables de control el tamaño de las empresas y el sector de actividad al que cada una pertenecía. Para medir el tamaño se utilizó el número de empleados de la empresa. La Tabla 4 muestra las medias, desviaciones típicas y correlaciones para las variables implicadas en el estudio. 
Tabla 4: Media, desviación típica y correlaciones

\begin{tabular}{lccccc}
\hline Variable & $\mathrm{N}$ & Media & d.t. & 1 & 2 \\
\hline 1. Resultados & 236 & 4,00 & 1,99 & & \\
\hline 2. Prácticas de recursos humanos de alto compromiso & 250 & 0,00 & 1,00 & $0,32 * * *$ & \\
\hline 3. Tamaño & 253 & 5,68 & 1,31 & 0,06 & $-0,12 *$ \\
\hline $\mathrm{p}<0,10 ; * * * \mathrm{p}<0,01$ & & & & &
\end{tabular}

\section{RESULTADOS}

El propósito planteado en esta investigación es analizar la influencia que ejercen las políticas de recursos humanos de alto compromiso o alta participación sobre los resultados corporativos de una muestra de empresas españolas. Con el fin de contrastar la hipótesis propuesta se realizó un análisis de regresión jerárquico, tal y como muestra la Tabla 5. El primer modelo de la tabla incluye únicamente las variables de control, mientras que el segundo añade a las anteriores las prácticas de recursos humanos de alto compromiso.

Tabla 5: Resultados del análisis de regresión jerárquico

\begin{tabular}{|c|c|c|c|c|}
\hline \multirow[t]{2}{*}{ Variables } & \multicolumn{2}{|c|}{ Modelo 1} & \multicolumn{2}{|c|}{ Modelo 2} \\
\hline & b & s.e. & $\mathrm{b}$ & s.e. \\
\hline Constante & 4,181 & 1,105 & 3,590 & 1,041 \\
\hline Tamaño & 0,113 & 0,122 & 0,038 & 0,115 \\
\hline Sector sic 20 & $-0,635$ & 0,939 & $-1,290$ & 0,88 \\
\hline Sector sic 22 & 0,892 & 1,156 & $-0,034$ & 1,096 \\
\hline Sector sic23 & 0,956 & 1,671 & 0,617 & 1,566 \\
\hline Sector sic 24 & $-2,764 *$ & 1,650 & $-3,540 * *$ & 1,553 \\
\hline Sector sic26 & 0,702 & 1,323 & 0,074 & 1,245 \\
\hline Sector sic 27 & $-1,090$ & 1,125 & $-2,316 * *$ & 1,078 \\
\hline Sector sic 28 & $-0,916$ & 0,945 & $-1,802 * *$ & 0,900 \\
\hline Sector sic 29 & 0,065 & 2,165 & $-1,850$ & 2,059 \\
\hline Sector sic 30 & $-1,381$ & 1,100 & $-2,311 * *$ & 1,045 \\
\hline Sector sic 31 & $-0,316$ & 1,650 & $-0,305$ & 1,546 \\
\hline Sector sic 32 & $-0,629$ & 1,003 & $-1,186$ & 0,946 \\
\hline Sector sic 33 & $-2,289^{* *}$ & 1,131 & $-2,401 * *$ & 1,060 \\
\hline Sector sic34 & $-0,584$ & 1,020 & $-1,285$ & 0,964 \\
\hline Sector sic 35 & 0,512 & 0,971 & $-1,505$ & 0,928 \\
\hline Sector sic 36 & $-0,953$ & 0,992 & $-1,924 * *$ & 0,947 \\
\hline Sector sic37 & $-1,429$ & 0,981 & $-2,262 * *$ & 0,932 \\
\hline Sector sic 38 & $-0,387$ & 1,198 & $-1,415$ & 1,138 \\
\hline \multicolumn{3}{|c|}{ Prácticas de recursos humanos de alto compromiso } & \multicolumn{2}{|l|}{$0,501 * * *$} \\
\hline $\mathrm{R}^{2}$ & 0,10 & \multicolumn{3}{|c|}{0,21} \\
\hline $\mathrm{F}$ & 1,216 & \multicolumn{3}{|c|}{$2,861 * * *$} \\
\hline $\mathrm{N}$ & 258 & \multicolumn{3}{|c|}{258} \\
\hline
\end{tabular}


La incorporación de las prácticas de recursos humanos de alto compromiso supuso un incremento de la varianza de un $11 \%$. De esta forma, el modelo 2 explica aproximadamente el $21 \%$ de la varianza de los resultados de las empresas de la muestra $(F=2,86 ; p<0,01)$. Los resultados permiten aseverar que las prácticas de recursos humanos denominadas de alta participación o alto compromiso influyen positivamente sobre los resultados corporativos, ya que el parámetro estimado de esa variable resultó ser positivo y estadísticamente significativo, lo que permite confirmar la hipótesis $\mathrm{H}$.

Respecto a las variables de control se encontraron algunas diferencias en los resultados de las empresas en función del sector de actividad al que pertenecían.

\section{CONCLUSIONES}

En este trabajo se ha comprobado como la forma de dirigir los recursos humanos tiene influencia sobre el éxito que alcanza la empresa. Los resultados obtenidos respaldan la hipótesis de que las empresas que utilizan un conjunto de prácticas de recursos humanos de alto compromiso obtienen mejores resultados que aquellas organizaciones que no las emplean. Basados en una muestra de empresas españolas, estos resultados están en consonancia con aquellos obtenidos en trabajos precedentes (Arthur 1994; Huselid 1995; MacDuffie 1995; Koch y McGrath 1996; Delaney y Huselid 1996; Delery y Doty 1996; Ichniowski, Shaw y Prennushi 1997; Huselid y Becker 1997; Bae y Lawler 2000).

Sobre la base de este estudio y de otros anteriores (Lawler 1992; Levine 1995; Pfeffer 1998) los directivos de empresas pueden conocer la influencia positiva que las prácticas de recursos humanos de alto compromiso pueden ejercer sobre la eficacia organizativa. Este estudio apoya el argumento de que un mayor uso de estas prácticas está asociado con mayores resultados organizativos y se espera que tanto académicos como directivos de empresa encuentren en este estudio una contribución a la literatura sobre recursos humanos y resultados organizativos, especialmente para el caso español.

Adicionalmente, este trabajo de investigación presenta algunas limitaciones. Una de ellas, es que no tiene en cuenta la existencia de complementariedades entre las prácticas que componen la dirección de 
recursos humanos. Aunque en este trabajo se ha adoptado una perspectiva de sistema y por tanto, no se ha examinado las prácticas de recursos humano de forma aislada e individual, el estudio de la forma en que esas prácticas se interrelacionan entre sí y cómo ello afecta a los resultados de la empresa contribuiría a mejorar la presente investigación y por tanto, constituye una línea de investigación a seguir en el futuro.

\section{BIBLIOGRAFÍA}

APRIL CHANG, W.J. and T. CHUN HUANG (2005) "Relationship between Strategic Human Resource Management and Firm Performance: A Contingency Perspective", International Journal of Manpower, Vol. 26, 5, pp. 434449.

ARTHUR, J. (1992) "The Link between Business Strategy and Industrial Relations Systems in American Steel Minimills", Industrial and Labour Relations Review, Vol. 45, pp. 488-506.

- (1994) "Effects of Human Resource Systems on Manufacturing Performance and Turnover", Academy of Management Journal, Vol. 37, pp. 670-687.

BAE, J. and J.J. LAWLER (2000) "Organizational and HRM Strategies in Korea: Impact on Firm Performance in an Emerging Economy", Academy of Management Journal, Vol. 43, pp. 502-517.

BALINGA, B.R.; R.C. MOYER and R.S. RAO (1996) "CEO Duality and Firm Performance: what's the Fuss?", Strategic Management Journal, Vol. 17, pp. 41-43.

BARNEY, J.B. and P.M. WRIGHT (1998) "On Becoming a Strategic Partner: The Role of Human Resources in gaining Competitive Advantage", Human Resource Management, Vol. 7, 1, pp. 31-46.

BARTEL, A.P. (1994) "Productivity Gains from the Implementation of Employee Training Programs", Industrial Relations, Vol. 33, pp. 411-425.

BECKER, B.E. and M.A. HUSELID (1998) "High Performance Work Systems and Firm Performance: A Synthesis of Research and Managerial Implications", Research in Personnel and Human Resources Journal, Vol. 16, 1, pp. 53-101.

CHEN, L.H.; S.Y. LIAW and T.Z. LeE (2003) "Using an HRM Pattern Approach to Examine the Productivity of Manufacturing Firms: An Empirical Study", International Journal of Manpower, Vol. 24, 3, pp. 299-318. 
CUtCheR-Gershenfeld, J.C. (1991) "The Impact on Economic Performance of a Transformation in Workplace Relations", Industrial and Labour Relations Review, Vol. 44, pp. 241-260.

DATTA, D.K.; J.P. GUTHRIE and P.M. WRIGHT (2005) "Human Resource Management and Labor Productivity: Does Industry Matter", Academy of Management Journal, Vol. 48, pp. 135-145.

DelaneY, J.T. and M.A. HuSELID (1996) "The Impact of Human Resource Management Practices on Perceptions of Organizational Performance", Academy of Management Journal, Vol. 39, pp. 949-969.

DELERY, J.E. and D.H. DOTY (1996) "Modes of Theorizing in Strategic Human Resource Management: Tests of Universalistic, Contingency and Configurational Performance Predictions", Academy of Management Journal, Vol. 39, pp. 802-835.

- and J.D. SHAW (2001) "The Strategic Management of People in Work Organizations: Review, Synthesis and Extension". K.M. RoWLAND and G.R. FERRIS (Eds.) Research in Personnel and Human Resource Management. Greenwich, CT: JAI Press, pp. 165-197.

DUNS AND BRADSTREET ESPAÑA (2001) Duns 50.000 Principales Empresas Españolas, Madrid.

GERHART, B. (1999) "Human Resource Management and Firm Performance: Measuring Issues and their Effect on Causal and Policy Inferences". P.M. WRIGHT, L.D. DYER, J.W. BOUdREAU and G.T. MILKOVICH (Eds.) Research in Personnel and Human Resource Management. Greenwich, CT: JAI Press, Supplement 4, pp. 31-51.

GUTHRIE, J.P. (2001) "High Involvement Work Practices, Turnover and Productivity: Evidence from New Zeland", Academy of Management Journal, Vol. 44, pp. 180-191.

HILL, C.W.L.; M.A. HITT and R.E. HoSkISSON (1992) "Cooperative versus Competitive Structures in Related and Unrelated Diversified Firms", Organization Science, Vol. 3, 4, pp. 501-521.

HOLZER, H.J. (1987) "Hiring Procedures in the Firm: Their Economic Determinants and Outcomes". M.M. KLEINER, R.N. BLOCK, M. ROOMKIN \& S.W. SAlSBURG (Eds.) Human Resources and the Performance of the Firm. Washington, DC: BNA Press.

HusELID, M.A. (1995) "The Impact of Human Resource Management Practices on Turnover, Productivity, and Corporate Financial Performance", Academy of Management Journal, Vol. 38, pp. 635-670. 
- and B.E. BECKER (1997) "The Impact of High Performance Work Systems, Implementation Effectiveness, and Alignment with Strategy on Shareholder Wealth", Unpublished paper, New Brunswick, NJ: Rutgers University.

ICHNIOWSKI, C.; K. SHAW and G. PRENNUSHI (1997) "The Effects of Human Resource Management Practices on Productivity: A Study of Steel Finishing Lines", American Economic Review, Vol. 87, pp. 291-313.

KATZ, H.C.; T.A. KOCHAN and K.R. GOBEILle (1983) "Industrial Relations Performance, Economic Performance and QWL Programs: An Interplant Analysis", Industrial and Labour Relations Review, Vol. 37, pp. 3-17.

KING, A.W. and C.P. ZeithamL (2001) "Competences and the Firm Performance: Examining the Causal Ambiguity Paradox", Strategic Management Journal, Vol. 22, pp. 75-99.

KoCH, M.J. and R.G. MCGRATH (1996) "Improving Labour Productivity: Human Resource Management Policies do Matter", Strategic Management Journal, Vol. 17, pp. 335-354.

KoCHAN, T.A. and P. OSTERMAN (1994) The Mutual Gains Enterprise. Boston, MA: Harvard Business School Press.

LAWLER, E. (1992) The Ultimate Advantage: Creating the High-Involvement Organization. SF: Jossey-Bass.

LEVINE, D. (1995) Reinventing the Workplace: How Business and Employers can Both Win. Brookings Institution Washington, DC.

MACDUFFIE, J.P. (1995) "Human Resource Bundles and Manufacturing: Performance: Organizational Logic and Flexible Production Systems in the World Auto Industry", Industrial and Labour Relations Review, Vol. 48, pp. 197-221.

MCCartney, J. and P. Teague (2004) "The Diffusion of High Performance Employment Practices in the Republic of Ireland", International Journal of Manpower, Vol. 25, 7, pp. 598-617.

MORISHIMA, M. (1991) "Information Sharing and Firm Performance in Japan", Industrial Relations, Vol. 30, pp. 37-61.

NAMAN, J.L. and D.P. SLEVIN (1993) "Entrepreneurship and the Concept of Fit: A Model and Empirical Tests", Strategic Management Journal, Vol. 14, pp. 137-153.

Pfeffer, J. (1994) "Competitive Advantage Trough People", California Management Review, Vol. 36, pp. 9-29.

- (1998) The Human Equation: Building Profits by Putting People First. Boston, MA: Harvard Business School Press. 
PODSAKOFF, P. and D. ORGAN (1986) "Self-Reports in Organizational Research: Problems and Prospects, Journal of Management, Vol. 12, pp. 531-544.

POWELL, T.C. (1995) "Total Quality Management as Competitive Advantage: A Review and Empirical Study", Strategic Management Journal, Vol. 16, pp. 15-37.

RICHARD, O.C. and N.B. JOHNSON (2001) "Strategic Human Resource Management Effectiveness and Firm Performance", International Journal of Human Resource Management, Vol. 12, pp. 299-310.

RoBBINS, S.P. (1990) Organization Theory: Structure, Designs and Applications, Prentice Hall International Editions, $3^{\text {a }}$ Edition, Englewood Cliffs.

Venkatraman, N. and V. Ramanujan (1986) "Measurement of Business Performance in Strategy Research: A Comparison of Approaches", Academy of Management Review, Vol. 11, 4, pp. 801-814.

- and - (1987) "Measurement of Business Economic Performance: An Examination of Method Convergence", Journal of Management, Vol. 13, 1, pp. 109-122.

WALton, R. (1985) "From 'Control' to Commitment in the Workplace", Harvard Business Review, Vol. 73, 2, pp. 77-84.

Welbourne, T.M. and A.O. AndReWs (1996) "Predicting the Performance of Initial Public Offerings: Should Human Resource Management be in the Equation?", Academy of Management Journal, Vol. 39, pp. 891-919.

WHITENER, E.M. (2001) "Do 'High Commitment' Human Resource Practices Affect Employee Commitment? A Cross-Level Analysis using Hierarchical Linear Modelling", Journal of Management, Vol. 27, pp. 515-535.

WieRSEMA, M.F. and K.A. BANTEL (1993) "Top Management Team Turnover as an Adaptation Mechanism: The Role of the Environment", Strategic Management Journal, Vol. 14, pp. 485-504.

WRIGHT, P.M. and G.C. MCMAHAN (1992) "Theoretical Perspectives for Human Resource Management", Journal of Management, Vol. 18, pp. 295-320.

-, - and A. MCWILLIAMS (1994) "Human Resources and Sustained Competitive Advantage: A Resource-Based Perspective", International Journal of Human Resource Management, Vol. 5, pp. 301-326.

YoundT, M.; S. SNeLL, J. DEAN and D. LePAK (1996) "Human Resource Management, Manufacturing Strategy, and Firm Performance", Academy of Management Journal, Vol. 39, pp. 836-886. 


\section{ANEXO 1. Indicadores incluidos en el cuestionario}

\begin{tabular}{|c|c|}
\hline VARIABLE & MEDIDA \\
\hline $\begin{array}{l}\text { Prácticas de } \\
\text { recursos humanos } \\
\alpha=0.90\end{array}$ & 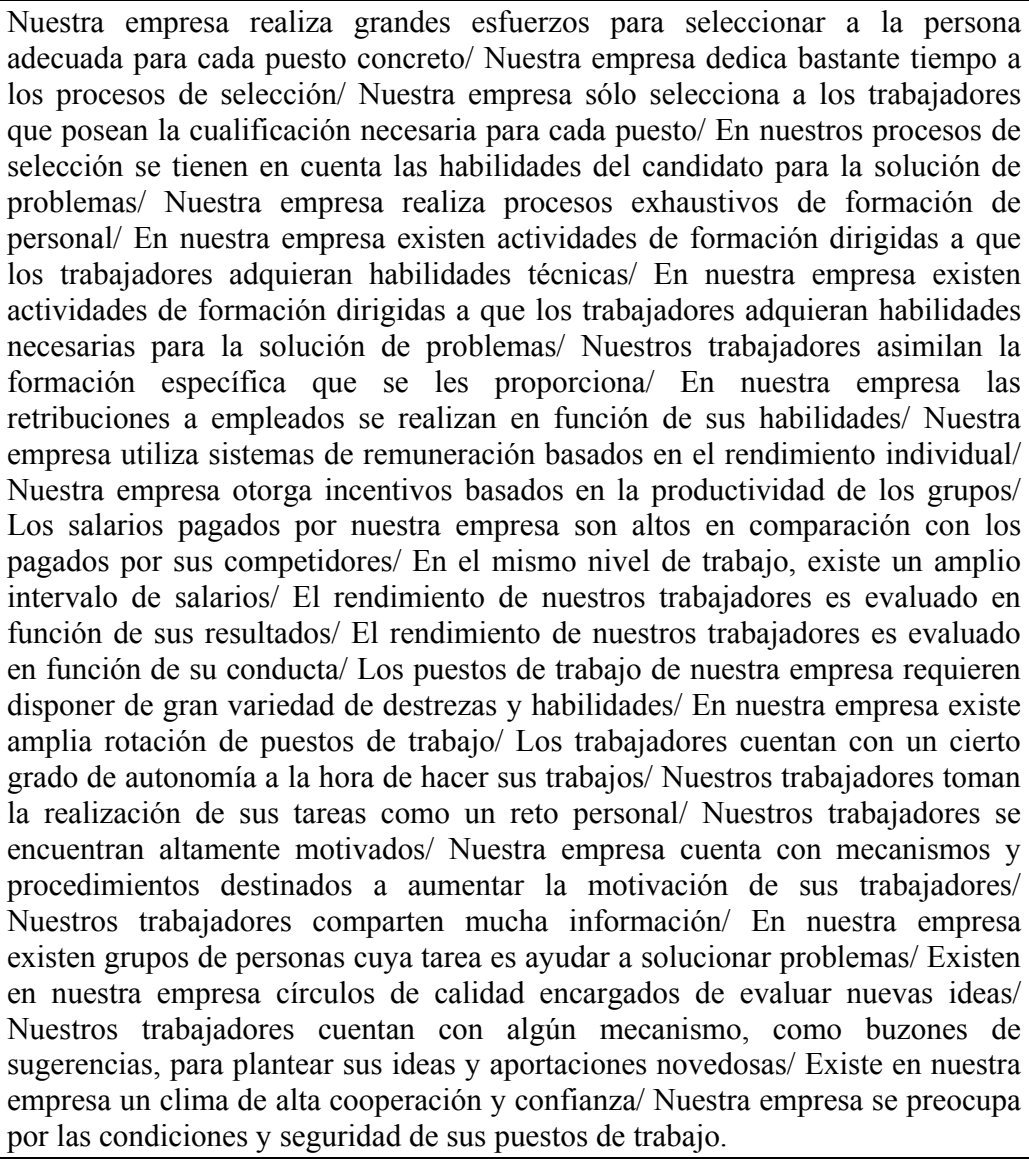 \\
\hline Resultados & $\begin{array}{l}\text { - Beneficio de explotación/ Crecimiento de las ventas/ Crecimiento de los } \\
\text { beneficios/ Cuota de mercado/ Rentabilidad sobre la inversión/ Desarrollo de } \\
\text { nuevos productos/ Desarrollo de mercados/ Nivel de conflictividad en la } \\
\text { empresa/ Productividad } \\
\text { - ROA }\end{array}$ \\
\hline
\end{tabular}

